\title{
A Novel Approach for Preparing Red Phosphorus Microcapsule by Hydrothermal Method
}

\author{
Yajuan Zhao (Corresponding author) \\ College of Chemistry and Materials Engineering, Wenzhou University \\ Wenzhou, Zhejiang, 325035, China \\ E-mail: zyj@wzu.edu.cn \\ Xing Huang \\ College of Chemistry and Materials Engineering, Wenzhou University \\ Wenzhou, Zhejiang, 325035, China \\ E-mail: huangxing27@126.com \\ Yulei Tai \\ College of Chemistry and Materials Engineering, Wenzhou University \\ Wenzhou, Zhejiang, 325035, China \\ E-mail: taiyulei@163.com \\ Daofa He \\ College of Chemistry and Materials Engineering, Wenzhou University \\ Wenzhou, Zhejiang, 325035, China \\ E-mail: hedaofa@wzu.edu.cn
}

The research is financed by the Science \& Technological Project of Zhejiang, China (Grant No.2009C33059) and the Science \& Technological Project of Wenzhou, China (Grant No.G20090146 and G20090148)

\begin{abstract}
A novel and effective protocol for the surface modification of the red phosphorus microcapsule via interfacial polymerization using hydrothermal method is reported. Composite microcapsules consisting of polymer-coated red phosphorous were prepared by interfacial polymerization of isocyanate and ethylenediamine in hydrothermal synthesis vessel. The morphology and magnetic property of microcapsules were characterized by scanning electron microscopy (SEM) and thermogravimetric analysis (TGA). Meanwhile, the water absorption and particle size distribution were examined. The relationship between the content of dispersants and the particle size of microencapsulated red phosphorus was investigated. On the basis of the experimental results, it seemed reasonable to conclude that the smaller the particle size of red phosphorus microcapsule was the better dispersibility in polymer, and then the higher flame retardant capability of polymer.
\end{abstract}

Keywords: Microcapsule, Red phosphorus, Polyurethane

\section{Introduction}

It is well known that red phosphorus (RP) is an inexpensive flame retardant with excellent performance(F. Laoutid et al., 2003;Wang Guo-An et al., 2006; Michael Pecht et al., 2006;Du Longchao et al., 2006; Hatsuhiko Harashina et al., 2006). However, recent reports have acknowledged the shortcoming that the red phosphorus can absorb moisture and release $\mathrm{PH}_{3}$ easily, because of its characteristic of inorganic matter. As a result, the application of red phosphorus has been limited. Currently, new challenges are being imposed on surface modification to satisfy the rising demand for safety of red phosphorus by encapsulated red phosphorus that should not only exhibit reinforced properties, but also improve security in multiple applications(Wang Haitao et al., 2008; Tsung-Han Ho et al., 2006; Liu Yuan et al., 2006; Wang Zhengzhou et al., 2002). In 
general, some inorganic matters and polymers (such as aluminum hydroxide, melamine resin, phenolic resin etc.) are used as shell to fabricate red phosphorus microcapsules (RPM). Also, hybrid mircrocapsules have been prepared by synthesis of red phosphorus in the interior of microcapsule, and form single or multiple layers. However, there are still some challenges on using these shell materials. For instance, it is still difficulty to disperse red phosphorus in inorganic materials such as aluminum hydroxide. Additionally, formaldehyde may be released from microcapsules used melamine resin and phenolic resin as shell. Therefore, in this work, we describe a novel route to preparing microcapsule for polyurethane resin as shell, which can form compact membrane and possess superior compatibility with other polymers. To date, a number of routes have been developed, but they produce microcapsules that cause the RP particles gathered easily and lead RPM oversize. Such problem would hinder their applications, because of the smaller of particle size of red phosphorus microcapsule, the better of dispersibility in polymer, and then the higher of flame retardant of polymer. Hence, the aim of this work is to develop a new protocol for the surface modification and the decrease of aggregate of RP particles via hydrothermal methods. To our knowledge, this is the first time that, the isocyanate and ethylenediamine are use for the fabratication of the RP microcapsule with polyurethane as shell through interfacial polymerization. In this process, dispersants are frequently utilized in the encapsulation of RP, such as sodium stearate (SS). More specifically, we show the relevance of the kinds of dispersants and the size of RPM particles, and the properties of RPM are measured by DSC-TAG and SEM, and the particle size of RPM is analyzed by laser particle-size analyzer.

\section{Experimental Section}

\subsection{Materials}

Red phosphorus powder was provided by Chemical Reagent Co.Tianjin(China), 200 mesh. 4,4'-diphenyl-methandiisocyanate (MDI) was purchased from Yantai Wanhua Polyurethanes Co. Ltd and ethylenediamine was purchased from Chenbei Fine Chemicals Company. All the other chemicals were of the highest purity available and were used as received.

\subsection{Instruments}

The $50 \mathrm{ml}$ of hydrothermal synthesis vessel with pressure gage was used for microencapsulation of RP. The stability was observed by Q600 integrated thermal analyzer, and was purchased from TA Company, U.S.A. The particle sizes were measured with STDY-S digital biological microscope image analyzer, and was provided by Shenzhen Kailida Science and Technology Co., Ltd. The morphology of the microcapsule was obtained on scanning electronic microscope (SEM; JSM-6700F), and was bought in Japan JEOL. The average grain diameter was measured with N4plus laser particle-size analyzer, and was purchased from Beckman Coulter, Inc. U.S.A. The fire resistance of red phosphorus microcapsule was carried out on a XYZ-460 oxygen index determinator, and was purchased from of Beijing east Co., Ltd.

\subsection{Preparation of RP Microcapsules}

RP microcapsules were synthesized by interfacial polymerization. Briefly, The $40 \mathrm{~mL}$ mixture, consisting of certain amount of pretreated red phosphorus, water, dispersant, ethylenediamine and organic solvent, was added into a $50 \mathrm{ml}$ hydrothermal synthesis vessel with pressure gage. The mixture was dried by oven $24 \mathrm{~h}$ at $423 \mathrm{~K}$. After the reaction temperature was slowly cooled to room temperature, MDI was added into hydrothermal synthesis vessel, and maintained at that temperature for $3 \mathrm{~h}$ with stirring. The mixture was then filtered, washed and desiccated to afford red phosphorus. The preparation of RPM was shown in the following Fig.1.

\section{Results and Discussion}

\subsection{Effect of different dispersant on size of RPM}

First, the different types of dispersants were studied, taking into account the fact that the grain diameter of microencapsulated red phosphorus would change with varying dispersants. A water solution of red phosphorus $(0.1 \mathrm{M})$ and dispersant $(0.01 \mathrm{M})$ and ethylenediamine $(0.05 \mathrm{M})$ was prepared by heating it at $423 \mathrm{~K}$ for $24 \mathrm{~h}$ in hydrothermal synthesis vessel with magnetic stirring. Three series dispersants, $\mathrm{D}_{1}$ (polyvinyl alcohol(PVA) and polyethylene glycol(PEG)), $\mathrm{D}_{2}$ (OP-10 and span-20 and span-60 and span-80), $\mathrm{D}_{3}$ (sodium laurylsulfate (SDS) and sodium dodecyl benzene sulfonate(SDBS) and sodium stearate(SS)) were added to hydrothermal synthesis vessel, respectively. Finally, the toluene solution of 4,4'-diphenyl-methandiisocyan-

ate $(\mathrm{MDI})(0.05 \mathrm{M})$ was added, and proceed for $3 \mathrm{~h}$. Microencapsulated red phosphorus was obtained by filter and the particle size distribution was measured by laser particle size analyzer. The grain diameter of microencapsulated red phosphorus preparing by three series dispersants respectively were shown in Fig. 2. Fig.2. presents the dependence of the grain diameter of microcapsule on the types of dispersants. It is well known that 
the polarity of dispersant affect the size distribution. The RP can be dispersed in polymer matrix easily by using $\mathrm{D}_{1}$ polymer dispersants, which are attributed to cationic characteristics. On the contrary, The RP cannot be dispersed in polymer matrix very well by using nonionic surfactant $D_{2}$ and anionic surfactant $D_{3}$. Compared with anionic surfactant $\mathrm{D}_{3}$, nonionic surfactant $\mathrm{D}_{2}$ is good dispersant for RP. But it does not always hold. The sodium stearate (SS) added in dispersant $\mathrm{D}_{3}$ obviously decrease the grain diameter of RP, which may be attributed to red phosphorus scattered by dispersants to micron scale at higher temperature and pressure. The result shows that the sodium stearate is the best dispersant in the process, due to electro-negativity interactions with surface of red phosphorus as an anionic surfactant. Although, SDBS and SDS are anionic surfactant, the hydrophobic chain is shorter than SS to lower surface activity.

\subsection{The moisture absorption rate and LOI of RP and RPM}

As we all know that the moisture absorption and LOI (limited oxygen index) of microencapsulated RP are directly related with the size of microcapsules, because smaller bare RP is easily coated and scattered in polymer. In Fig.3, we can find that bare RP and coated RP have the larger difference of moisture absorption rate. The content of RPM was achieved to $9 \%$, and the limited oxygen index was $27 \%$.

\subsection{Appearance on red phosphorus microcapsule}

The red phosphorus microcapsule is a kind of lavender and fluid powder in this paper. Content of red phosphorus $\geq 85 \%$, moisture absorption rate $\leq 0.3 \%$, ignition point $\geq 350^{\circ} \mathrm{C}, \mathrm{PH}_{3}<1 \mathrm{ppm}$. The uncoated red phosphorus is shown in Fig.4. The red phosphorus microcapsule is thousand-fold (1,000 times) zoomed-in aggregation, and is displayed in Fig.4 and Fig.5. The image of Fig.4 was measured by Model STDY-S digital biological microscope image analyzer. The red phosphorus microcapsule with small particle diameter is invisibly observed, moisture in the air is absorbed by uncoated red phosphorus and water trail is left around the red phosphorus.

In Fig. 5, the morphology of red phosphorus microcapsule is measured by scanning electronic microscope, showing that the red phosphorus has been coated with polyurethane resin. As lavender and fluid power, red phosphorus does not absorb moisture while being put in the air.

\subsection{Particle analysis of RPM}

The particle size and distribution (frequency analysis) of the red phosphorus microcapsule is presented in Fig.6, which is measured by laser particle size analyzer. The average particle diameter is $1,019 \mathrm{~nm}$, and is clearly showed by abundance curve, which is a example of particle size and distribution of RPM in this paper.

\subsection{Stability analysis of RPM}

As shown in Fig.7, comparing DSC-TGA spectrograms of RP and RPM, we can conclude that the thermo gravimetric curve at around $230^{\circ} \mathrm{C}$ has a gradient reduction, because of the decomposition of the polyurethane resin which is on the surface of the red phosphorus.

\section{Conclusion}

Red phosphorus is an innocuous, low-consumption, low-smoke and inexpensive flame retardant with excellent performance, which has extensive potential applications in many fields by surface modification. The microencapsulation of red phosphorus is one of the main modification methods. In this paper, A novel and effective protocol for the surface modification of the red phosphorus microcapsule via interfacial polymerization using hydrothermal method has been reported. polyurethane resin is used as the shell materials to prepare the red phosphorus microcapsules in hydrothermal synthesis vessel. The effects of dispersants on sizes of RPM are investigated. The results elucidate that the sodium stearate is the best dispersant, due to electro-negativity interactions with surface of red phosphorus as an anionic surfactant, the red phosphorus is coated by polyurethane resin successfully, and exhibit reasonable moisture absorption rate. Other surface treatment methods of red phosphorus still require further research.

\section{References}

Du Longchao, Qu Baojun and Xu Zhenjin. (2006). Flammability characteristics and synergistic effect of hydrotalcite with microencapsulated red phosphorus in halogen-free flame retardant EVA composite. Polymer Degradation and Stability, 91,995-1001.

F., Laoutid, L., Ferry, J.M., Lopez,Cuesta and A., Crespy. (2003). Red phosphorus/aluminium oxide compositions as flame retardants in recycled poly (ethylene terephthalate). Polymer Degradation and Stability, 82,357-363.

Hatsuhiko Harashina, Yoshihisa Tajima and Takahito Itoh. (2006). Synergistic effect of red phosphorus, novolac 
and melamine ternary combination on flame retardancy of poly(oxymethylene). Polymer Degradation and Stability, 91, 1996-2002.

Liu Yuan and Wang Qi. (2006). Melamine cyanurate-microencapsulated red phosphorus flame retardant unreinforced and glass fiber reinforced polyamide 66. Polymer Degradation and Stability, 91, 3103-3109.

Michael Pecht and Yuliang Deng. (2006). Electronic device encapsulation using red phosphorus flame retardants. Microelectronics Reliability, 46, 53-62.

Tsung-Han Ho, Tsu-Shang Leu and Yih-Min Sun. (2006). Thermal degradation kinetics and flame retardancy of phosphorus-containing dicyclopentadiene epoxy resins. Polymer Degradation and Stability, 91, 2347-2356.

Wang Guo-An, Wang Cheng-Chien and Chen Chun-Yung. (2006). The flame retardant material e 1. Studies on thermal characteristics and flame retardance behavior of phosphorus-containing copolymer of methyl methacrylate with 2-methacryloxyethyl phenyl phosphate. Polymer Degradation and Stability, 91, 2683-2690.

Wang Haitao, Meng Xiangfu and Wen Bin. (2008). A simple route for the preparation of red phosphorus microcapsule with fine particle distribution. Materials Letters, 62, 3745-3747.

Wang Zhengzhou, Wu Guosheng, Hu Yuan. (2002). Thermal degradation of magnesium hydroxide and red phosphorus flame retarded polyethylene composites. Polymer Degradation and Stability, 77, 427-434.

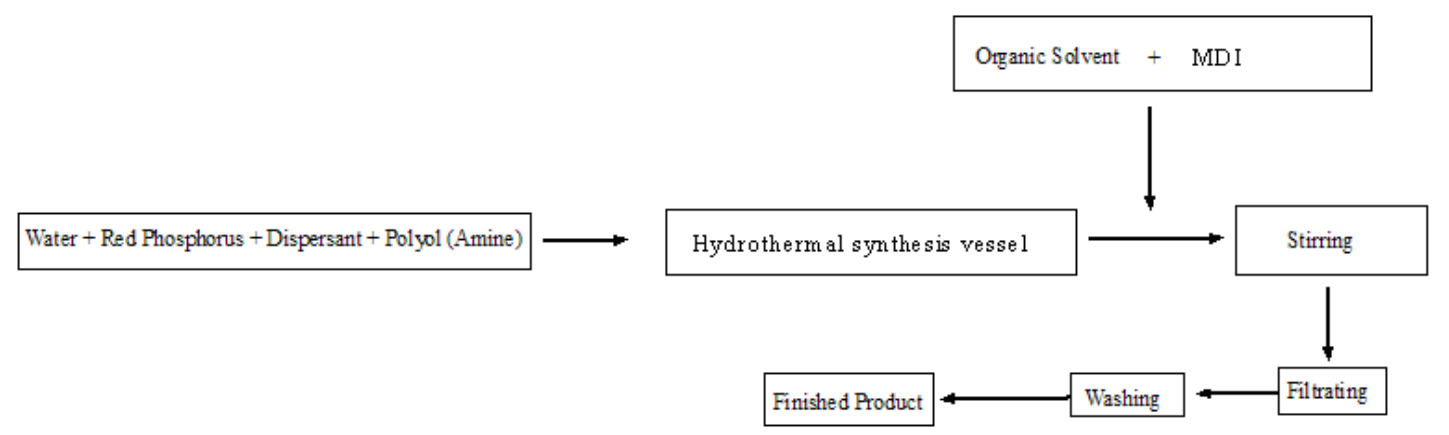

Figure 1. The Schematic of the preparation of RPM

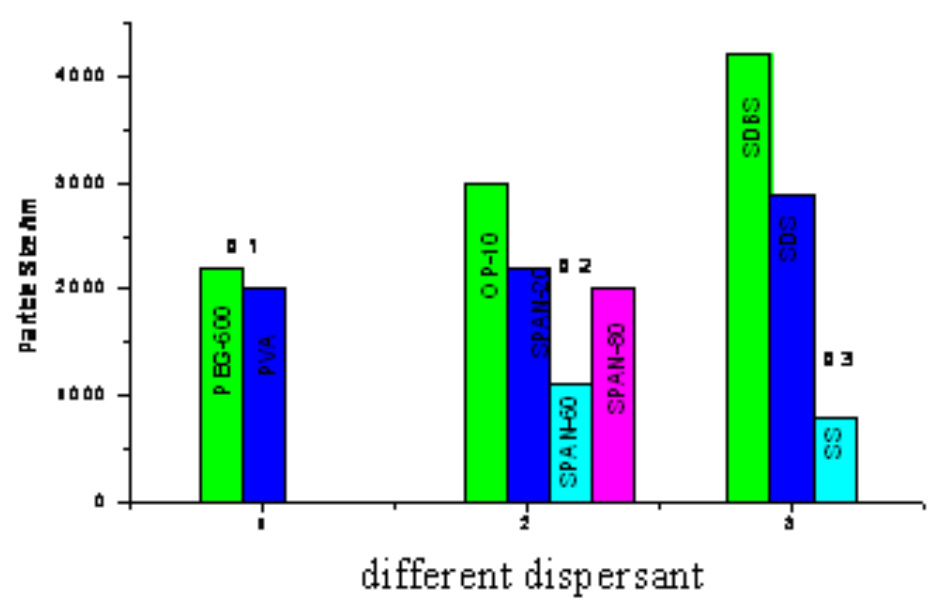

Figure 2. The effect of particle size on different dispersant 


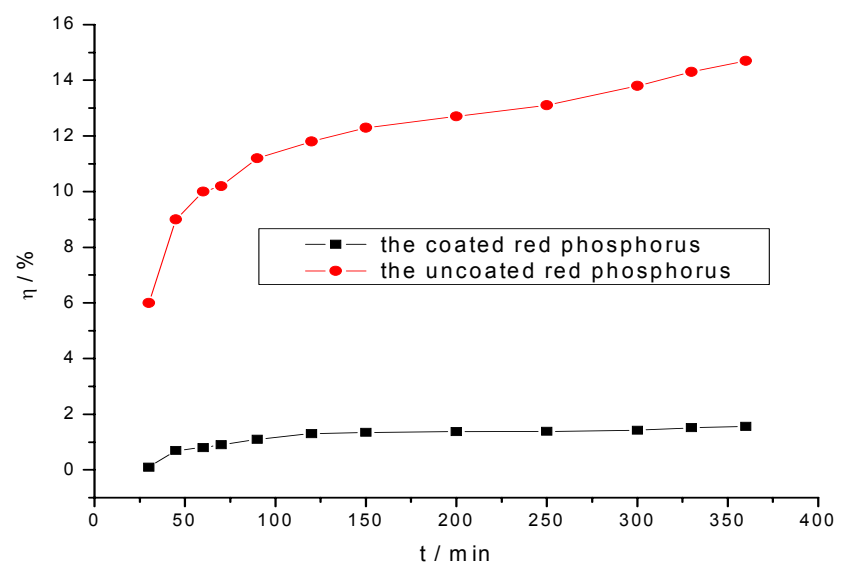

Figure 3. The moisture absorption rate for the uncoated and coated red phosphorus

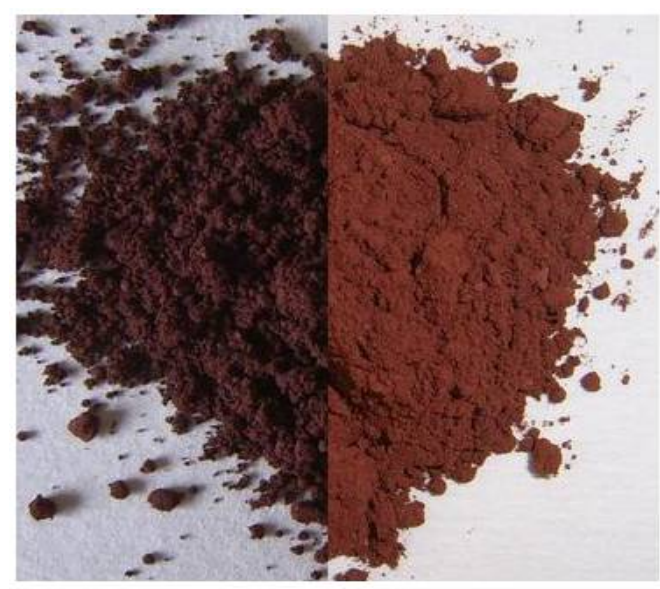

Figure 4. The figure of uncoated red phosphorus and red phosphorus microcapsule
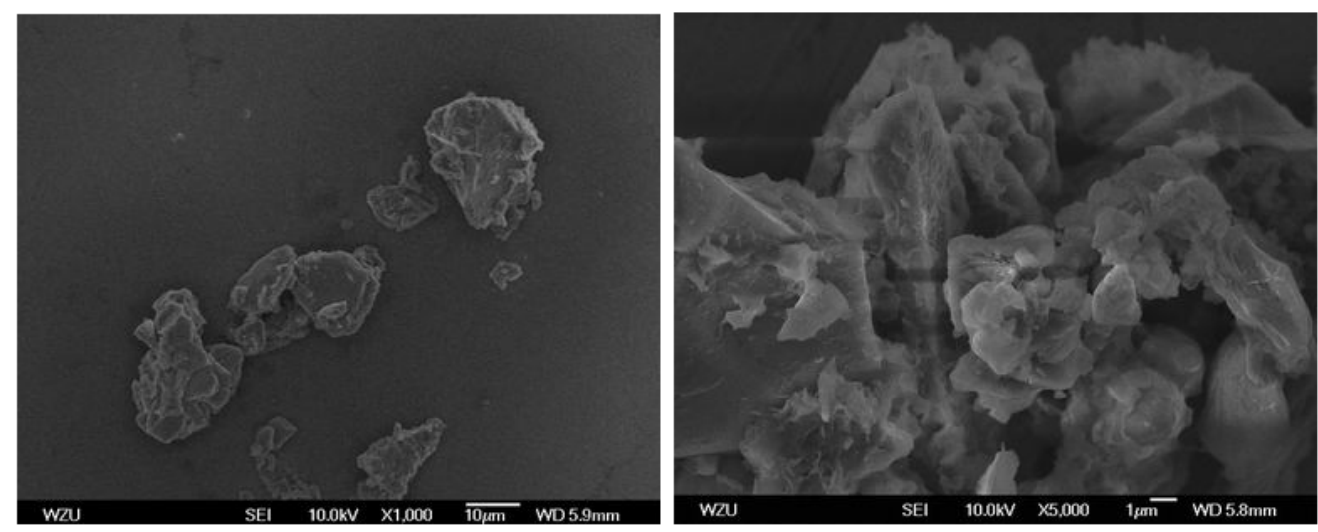

Figure 5. SEM of red phosphorus microcapsule 


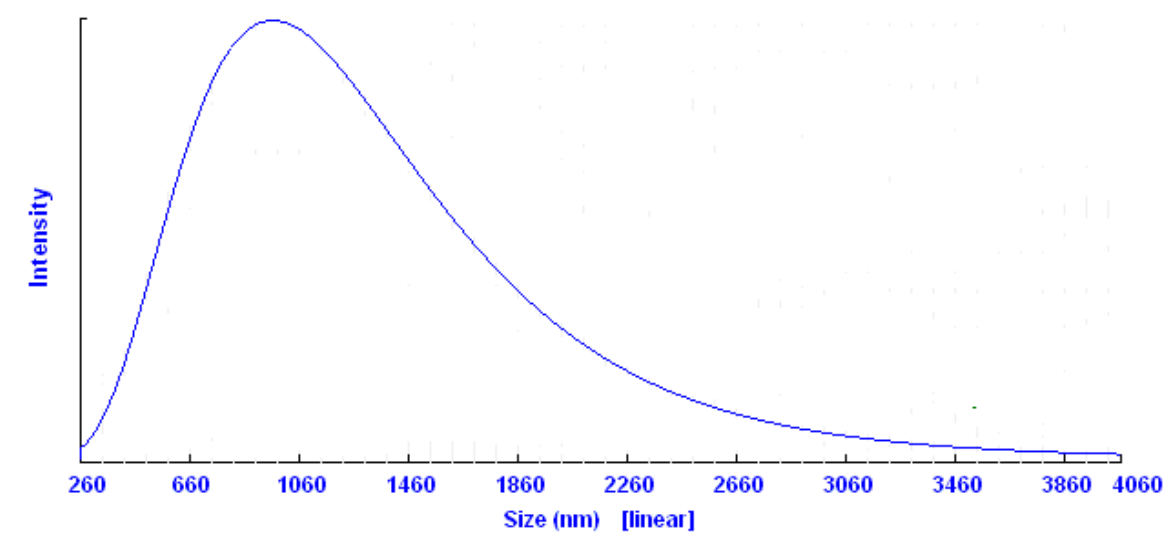

Figure 6. The particle size and distribution curve of RPM

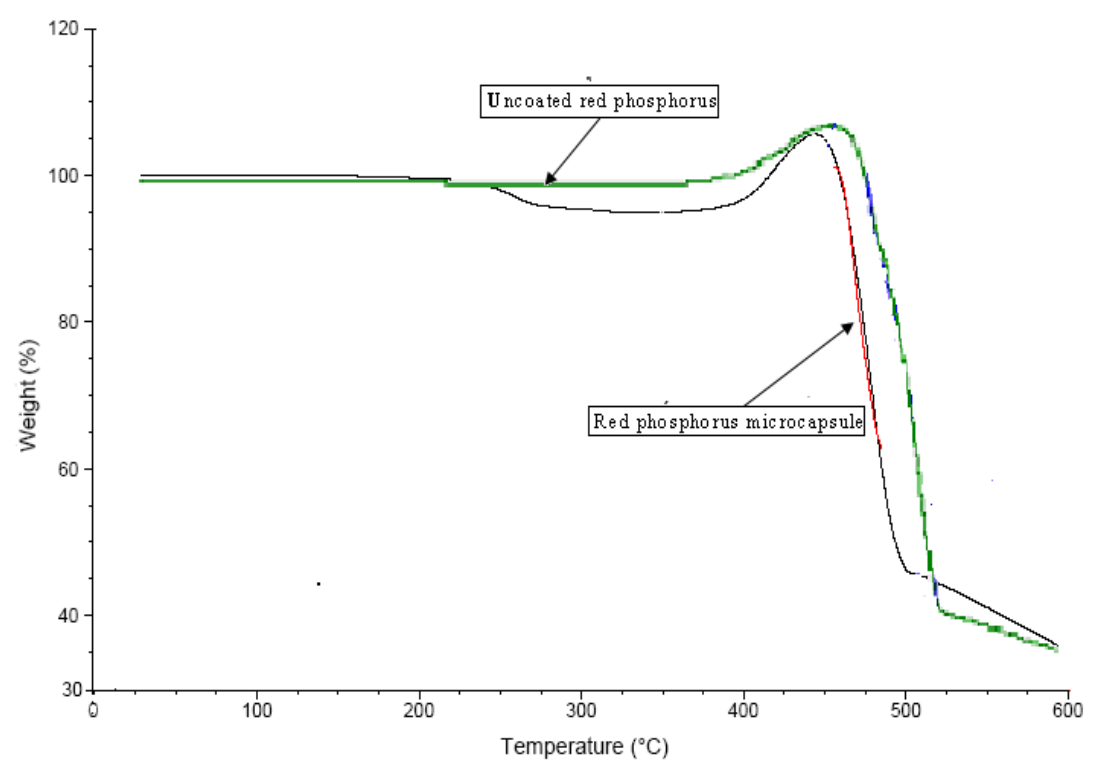

Figure 7. The DSC-TGA curve of RP and RPM 